ISSN (O) 2589-8779

(P) 2589-8760

CMRO 04 (03), 867-877 (2021)

REVIEW ARTICLE

\title{
Rosuvastatin and Fenofibrate Combination in The Treatment of Mixed Hyperlipidemia: A Narrative Review
}

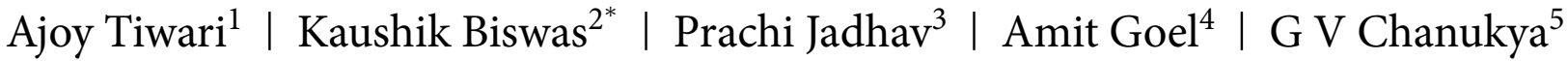

${ }^{1} \mathrm{MD}$, FICP, Consultant

Diabetologist. Jai Clinic, Lucknow

${ }^{2} \mathrm{MD}$, DM Endocrinology.

Consultant Endocrinologist.

SSKM Hospital, Kolkata

${ }^{3}$ MBBS, MD, DM Endocrinology. Department of Endocrinology, CPR Hospital, Kolhapur.

${ }^{4}$ DM Endocrinology. Consultant Endocrinologist. AG Centre for Diabetes Thyroid and Endocrine. Secunderabad

${ }^{5}$ MBBS. MD, DM. Consultant Endocrinologist, Dr Chanukya's Endocrine and Diabetes Centre, Hyderabad

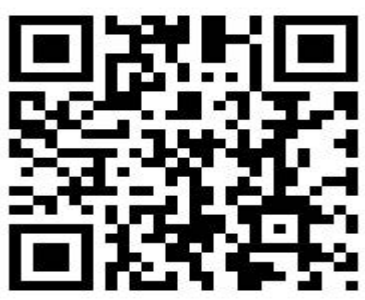

\section{Abstract}

Introduction: Patients with mixed dyslipidemia are presented with high levels of low-density lipid cholesterol (LDL-C), triglycerides (TG), and reduced high-density lipid cholesterol (HDL-C). Though useful in lowering LDL-C, therapy with rosuvastatin is insufficient in optimizing the overall lipid profile, thus putting the patient at risk of residual cardiovascular risk. A combination of statin with other lipidmodifying agents has been used with more efficient lipid control and cardiovascular risk prevention. Of these, fenofibric acid is the most frequently used, along with rosuvastatin.

Methods: Authors conducted a literature search of published literature to assess the use of rosuvastatin and fenofibrate combination in the management of mixed hyperlipidaemia.

Results and discussion: The authors selected a total of 46 articles to be included in the review. Due to the small number of articles and heterogeneity on the combination of rosuvastatin and fenofibrate combination in mixed hyperlipidemia, the findings herein are presented using narrative summaries. Based on the thorough assessment of the selected literature, the essential themes that emerged from the review include safety and efficacy of rosuvastatin and fenofibrate combination, place of therapy of rosuvastatin, and fenofibrate combination, and potential cardiovascular risk reduction with rosuvastatin and fenofibrate combination.

Conclusion: Based on the review, the authors suggested that the combination therapy with fenofibric acid was beneficial, well-tolerated with a similar safety profile compared with statin monotherapy. The combination therapy of moderate dose rosuvastatin and fenofibric acid led to a reduction of cardiovascular risk factors via several pathways. Keywords: Rosuvastatin, fenofibric acid, Mixed dyslipidemia, LDL-C, HDL-C, triglycerides

Copyright : (C) 2021 The Authors. Published by Publisher. This is an open access article under the CC BY-NC-ND license (https://creativecommons.org/licenses/by-nc-nd/4.0/). 


\section{1 | INTRODUCTION}

$\mathrm{T}$ he management of coronary heart disease has transformed with statin therapy as the reduction of low-density lipoprotein cholesterol (LDL-C) is an essential factor. Studies have shown that the use of statins leads to a $20-40 \%$ reduction in LDL-C levels, which further led to a 25 $35 \%$ lowering of the risk of myocardial infarction and stroke. Despite this protection, there remains a $65-75 \%$ risk of myocardial infarction and stroke in patients treated with statins $(1,2)$.

Currently, high LDL-C, triglycerides (TG), and low levels of high-density lipoprotein cholesterol (HDLC) are considered to be risk factors for coronary heart disease (CHD) (3). The significant features of patients with mixed dyslipidemia are raised TGs $(\geq 150 \mathrm{mg} / \mathrm{dL})$, low HDL-C ( $<40 \mathrm{mg} / \mathrm{dL}$ men, $<50$ $\mathrm{mg} / \mathrm{dL}$ women), and a moderate increase in LDL$\mathrm{C}$ with a high concentration of small LDL particles. The presence of this abnormal 'lipid triad,' along with abdominal obesity, impaired fasting glucose, and high blood pressure, leads to an increased risk for CHD in patients with mixed dyslipidemia $(4,5)$.

Hence, it becomes imperative to strategically use aggressive lipid-altering therapy to manage cardiovascular risk profiles in individuals with mixed dyslipidemia. In such cases, monotherapy, often with statins, is unable to achieve the targeted optimisation of increased LDL-C and TG levels along with the low levels of HDL-C. In such a situation, the use of combination therapy becomes imperative (6). The combination of statins with fibric acid derivatives is a commonly used therapy in individuals with mixed dyslipidemia (6). Rosuvastatin has demonstrated significant superiority over other statins used routinely (7) The STELLAR trial has depicted that rosuvastatin reduced non-HDL-C by $42.0 \%$ to $50.9 \%$ compared with $34.4 \%$ to $48.1 \%$ with atorvastatin, $26.0 \%$ to $41.8 \%$ with simvastatin, and $18.6 \%$ to $27.4 \%$ with pravastatin. Similarly, adult treatment panel III LDL cholesterol goals were achieved in 82 to $89 \%$ of patients treated with rosuvastatin (10 to 40 $\mathrm{mg}$ ), compared with 69 to $85 \%$ patients treated with $10 \mathrm{mg}$ to $80 \mathrm{mg}$ atorvastatin; rosuvastatin reduced apo B protein by $36.7 \%$ to $45.3 \%$ compared with $29.4 \%$ to $42.9 \%$ with atorvastatin, $22.2 \%$ to $34.7 \%$ with simvastatin, and $14.7 \%$ to $23.0 \%$ with pravastatin (8).

Fenofibric acid is frequently used in combination therapy with a statin. Both the drugs have different mechanisms of action and exhibit a complementary pharmacodynamic effect on lipid levels. Fibrates change the lipid levels via activation of peroxisome proliferator-activated receptors (PPARs), which regulate gene transcription. On the other hand, statins affect lipid levels by inhibiting 3-hydroxy3-methylglutaryl-coenzyme A (HMG-CoA) reductase (9).

In this article, the authors have attempted to throw insight on the place of therapy of rosuvastatin and fenofibrate combination therapy versus statin monotherapy, the safety profile of the combination, and its potential role in reducing the cardiovascular risk in patients of mixed dyslipidemia.

\section{2 | METHODS}

The authors conducted a detailed review of published literature to evaluate the place of therapy of rosuvastatin and fenofibrate combination therapy compared with statin therapy, safety, efficacy, and the reduction of cardiovascular side effects in patients with mixed hyperlipidemia.

A search was conducted on PubMed, Medline, and Google Scholar with the search terms (Rosuvastatin) AND (Fenofibrate) between 2003 and 2020. The author's goal was to analyze all the published literature comprising randomized controlled trials, clinical trials, retrospective and prospective research, systematic reviews, and meta-analysis for rosuvastatin and fenofibrate combination in managing mixed hyperlipidemia. The search returned 32 results, out

Supplementary information The online version of this article (https://doi.org/10.15520/jemro.v4i03.40 5) contains supplementary material, which is available to authorized users.

Corresponding Author: Kaushik Biswas

MD, DM Endocrinology. Consultant Endocrinologist. SSKM Hospital, Kolkata

Email: biswask09@gmail.com 


\section{ROSUVASTATIN AND FENOFIBRATE COMBINATION IN THE TREATMENT OF MIXED HYPERLIPIDEMIA: A NARRATIVE REVIEW}

of which 27 articles were screened and selected. Further, in a backward chronological search, the lists of all relevant articles were checked for citations that could not be identified in the primary search. Titles and abstracts from the electronic search were screened, and full-text articles meeting the selection criteria were obtained. The study data were extracted by reading the complete article.

Title and abstracts from the electronic search were checked, and full-text articles on the treatment of rosuvastatin and fenofibrate in mixed hyperlipidemia were obtained. Crucial information from all the articles was extracted. Two investigators independently extracted data from eligible studies, and any differences were resolved through discussion and consensus between the authors. Where an agreement was not reached, arbitration was done by the third author. The selected articles were then qualitatively analyzed by the investigators.

\section{3 | RESULTS AND DISCUSSION}

Based on the search, a total of 46 articles were selected to be included in the review. Due to the small number of articles and heterogeneity on the combination of rosuvastatin and fenofibrate combination in mixed hyperlipidemia, the findings herein are presented using narrative summaries. Based on the thorough assessment of the selected literature, the essential themes that emerged from the review include safety and efficacy of rosuvastatin and fenofibrate combination, place of therapy of rosuvastatin, and fenofibrate combination, and potential cardiovascular risk reduction with rosuvastatin and fenofibrate combination.

\section{Rosuvastatin-fenofibrate pharmacokinetic inter- actions}

In a multiple-dose, open-label, 3-period, randomized, cross-over design assessing the pharmacokinetic interaction between the two drugs, the authors suggested that they showed no clinically significant pharmacokinetic interaction between fenofibric acid at the full clinical dose and rosuvastatin at the highest approved dose. The findings of the above study demonstrated that co-administering the two drugs had no substantial effect on the steady-state $\mathrm{C}_{\min }$ or AUC 24 of rosuvastatin ( $\left.\mathrm{p}>.05\right)$, but the $\mathrm{C}_{\max }$ was found to be raised by $20 \%(99 \% \mathrm{CI}$ : $12 \%-18 \%$ ) (6). Similar results were obtained from another open-label, randomized, 3-way cross-over trial consisting of three 7-day treatment periods. The study findings showed that coadministration of rosuvastatin and fenofibrate produced minimal changes in rosuvastatin and fenofibric acid exposure about a minor increase in the AUC from 0 to 24 hours and $\mathrm{C}_{\max }$ of rosuvastatin; the respective geometric least-square means increased by $7 \%(90 \% \mathrm{CI}, 1.00$ $1.15)$ and $21 \%(90 \%$ CI, 1.14-1.28). The pharmacokinetic parameters of fenofibric acid were similar when fenofibrate was administered alone and with rosuvastatin: the geometric least-square mean for fenofibric acid AUC from 0 to 8 hours and $\mathrm{C}_{\max }$ decreased by $4 \%(90 \% \mathrm{CI}, 0.90-1.02)$ and $9 \%(90 \%$ CI, 0.84-1.00), respectively (10).

\section{Place of therapy of rosuvastatin and fenofibrate combination}

One of the key factors in preventing cardiovascular disease prevention is the reduction of low-density lipoprotein cholesterol, and it also forms the main aspect of hypolipidemic therapy. $(11,12)$. The treatment of the lipid triad calls for the use of combination therapy. Evidence has suggested that the concomitant use of statins and fibrates may lead to a positive effect on the lipid triad. The combination of rosuvastatin and fenofibrate assumes significance here, given the potency of rosuvastatin to lower LDL-C and fenofibrate's efficacy in reducing triglycerides. Rosuvastatin is known to reduce LDL-C by $45 \%-63 \%$ with doses of $5-20 \mathrm{mg}$ per day, a much higher mean reduction compared with equivalent doses of other statins (13). Fenofibric acid has an increased efficacy in reducing triglycerides and increasing HDL-C in patients on rosuvastatin therapy with a favorable safety profile. Rosuvastatin -fenofibric acid fixed-dose combinations have significantly improved triglycerides, HDL-C, nonHDL-C, apolipoprotein B, and high sensitivity Creactive protein levels compared with simvastatin monotherapy $(\mathrm{P} \leq .04$ for all comparisons) (14). The safety and efficacy studies have shown that there are no major adverse reactions following the use of combination and rosuvastatin, and fenofibrate is a 
safe combination to treat difficult to manage mixed dyslipidemia patients (13).

The 2018 AHA/ACC/AACVPR/AAPA/ABC/ACPM/AD Guideline on the management of blood cholesterol and the 2019 ESC/EAS guidelines for the management of dyslipidaemias recommend that it is safer to use fenofibrate with a statin since it has a lower risk of severe myopathy. It is vital to reduce triglycerides whenever levels exceed $500 \mathrm{mg} / \mathrm{dL}$ $(5.6 \mathrm{mmol} / \mathrm{L})$ to prevent pancreatitis in patients with severe hypertriglyceridemia. This can be achieved by prescribing a very low-fat diet and adding fibrates or omega-3 fatty acids for patients with persistently high severe hypertriglyceridemia $(15,16)$.

\section{Efficacy of rosuvastatin and fenofibrate in dia- betes}

The first open-label randomized study to directly compare the high doses of rosuvastatin $(40 \mathrm{mg})[\mathrm{R}]$ with low doses of statin $(10 \mathrm{mg})$ plus fenofibrate (200 mg) [RF] or $\Omega-3$ fatty acids (2 g) [RN] in the treatment of mixed hyperlipidemia showed that non-HDL-C levels were reduced in all groups: in $\mathrm{R}$ group by $54 \%$, in $\mathrm{RF}$ group by $42 \%$ and $\mathrm{RN}$ group by $42 \%$. Significant reductions in total cholesterol, LDL-C and triglyceride levels were observed in all groups. At the end of the 3-month treatment duration, there was a significant reduction of non-HDL-C in all treatment groups $(\mathrm{p}<0.001$ compared to baseline values) with a more significant decrease in the $R$ group ( $p<0.05$ compared to $\mathrm{RF}$ and $\mathrm{RN}$ groups). The proportion of patients reaching the non-HDL-C target value was significantly more in the $\mathrm{R}$ group than the RF group $(p<0.05)$ but not in the RN group. While the monotherapy was more effective in reducing non-HDL-C, the combination of rosuvastatin plus fenofibrate led to a more potent effect in lowering triglyceride levels and raising HDL-C values compared with the other two treatment groups (LDLC: R group-304 \pm 69 to $164 \pm 37$, Group RF-300 \pm 45 to $197 \pm 40$, Group RN-284 \pm 42 to $185 \pm 37$; HDL$\mathrm{C}$ : R group- $50 \pm 8$ to $52 \pm 8$, RF Group- $52 \pm 10$ to 56 \pm 12 , RN Group $48 \pm 10$ to $50 \pm 10$; non-HDL-C: R Group $253 \pm 60$ to $117 \pm 32$, RF Group $247 \pm 38$ to $141 \pm 39$, RN Group $235 \pm 38$ to $135 \pm 33$ ) (17).

A randomized trial showed that when a comparative assessment was made between the effect of high dose rosuvastatin monotherapy with moderate dosing combined with fenofibrate or $\omega-3$ fatty acids

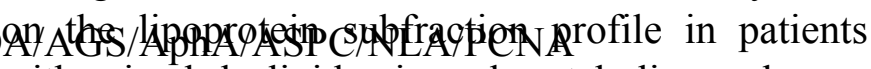
with mixed dyslipidemia and metabolic syndrome, rosuvastatin + fenofibrate was the most effective treatment. The findings of the study showed that the mean low-density lipoprotein and HDL-C level were significantly increased while insulin resistance was reduced (18). Similar results were seen when high doses of rosuvastatin $(40 \mathrm{mg})[\mathrm{R}]$ or a combination of low doses of rosuvastatin $(10 \mathrm{mg}$ ) with fenofibrate (200 mg) [RF] were proven to be superior to the rosuvastatin $(10 \mathrm{mg})$-Omega 3 fatty acid ( $2 \mathrm{~g})$ combination $[R \Omega]$ treatment given daily in reducing inflammatory indices. The HDL-LpPLA2 activity was raised more in the RF group ( $+43 \%)$ compared with the $\mathrm{R}$ and $\mathrm{R} \Omega$ groups $(+18 \%$ and $+35 \%$, respectively; $\mathrm{p}<0.05$ for both comparisons) (19).

It was seen in many comparative assessments that the use of rosuvastatin and fenofibric acid was more efficacious than rosuvastatin alone in patients with mixed dyslipidemia. In a post hoc analysis of patients with mixed dyslipidemia, the combination therapy compared with rosuvastatin monotherapy showed comparable effects in achieving risk-stratified lowdensity lipid-C goals. However, measures of total atherogenic burden were improved (20). In a study where 1-year therapy with rosuvastatin and fenofibric acid was conducted, the results showed the combination to be well-tolerated (21).

Current evidence suggests that statin/fibrate combination therapy is favorable in changing the lipid profile of T2Dm patients with high TG and low HDL-C profile, which is linked with increased cardiovascular risk (22). A post hoc analysis was done in trials led by Jones et al. and Rosenson et al., including type 2 diabetes patients with mixed dyslipidemia treated with rosuvastatin $(5,10$, or $20 \mathrm{mg})$ fenofibric acid $135 \mathrm{mg}$, or the combination of them for 12 weeks. The results revealed that the combination therapy with rosuvastatin and fenofibric acid led to a significantly higher proportion of type 2 diabetes mellitus patients achieving the individual and lipid targets than the corresponding monotherapies (23).

These results were consonant in patients with diabetes too, who, when treated with a combination of 


\section{ROSUVASTATIN AND FENOFIBRATE COMBINATION IN THE TREATMENT OF MIXED HYPERLIPIDEMIA: A NARRATIVE REVIEW}

rosuvastatin and fenofibric acid, achieved individual and combined lipid targets as compared with the corresponding dose of rosuvastatin monotherapy (24).

\section{Elderly patients}

Sub-analysis of two randomized controlled trials showed that the combination of rosuvastatin 5,10 , or $20 \mathrm{mg}$ with fenofibric acid $135 \mathrm{mg}$ improved the overall lipid profile when given to elderly patients (age 65 years or older) with mixed dyslipidemia. The study findings demonstrated that the combination therapy reduced LDL-C by $31.8 \%-47.2 \%$ vs. $10.6 \%$ with fenofibric acid monotherapy $(\mathrm{P}<0.001)$. Combination therapy also raised HDL-C by $21.9 \%$ $27.0 \%$ vs. $5.9 \%-9.9 \%$ with rosuvastatin monotherapy $(\mathrm{P}<0.001)$ and reduced triglycerides by $48.3 \%$ $53.5 \%$ vs. $20.7 \%-32.8 \%$ with rosuvastatin monotherapy $(\mathrm{P}<0.001)$. There were no new or unexpected safety issues observed with the combination therapy (25).

\section{Effects on biomarkers}

Another benefit of the combination therapy with rosuvastatin and fenofibric acid is the improvement in the inflammatory biomarker, high sensitivity $\mathrm{C}$ reactive protein (hsCRP), and other lipid abnormalities in patients with mixed dyslipidemia and high hsCRP. Increased levels of hsCRP are indicative of an increased risk of cardiovascular events. The post hoc analysis reported that $65 \%(1416 / 2182)$ of patients had pre-treatment baseline hsCRP $\geq 2 \mathrm{mg} / \mathrm{L}$. Among all treatment groups, groups, higher reduction in hsCRP was seen in patients with greater baseline hsCRP; however, improvements in other lipids/apolipoprotein were comparable between the baseline hsCRP categories. In patients with high hsCRP ( $\geq 2 \mathrm{mg} / \mathrm{L})$ remaining after 12 weeks of rosuvastatin 10,20 , or $40 \mathrm{mg}$ monotherapy, hsCRP was lowered by $\sim 36 \%$ after changing to rosuvastatin $20 \mathrm{mg}$ and fenofibric acid $135 \mathrm{mg}$ for up to 52 weeks, and $\sim 36$ of patients shifted from hsCRP $\geq 2 \mathrm{mg} / \mathrm{L}$ to $<2 \mathrm{mg} / \mathrm{L}$. Thus, combination therapy with rosuvastatin and fibrate is more effective than statin monotherapy for reducing hsCRP and hence a potential treatment option for patients with several lipid risk factors (26).

Rosuvastatin has shown a beneficial effect on oxidative stress markers, while fenofibrate is associated with reduced oxidative stress. In a randomized, openlabel, blinded endpoint (PROBE) study, patients with mixed dyslipidemia on a standard statin dose on uncontrolled lipid targets were randomly selected to transition to a higher dose of rosuvastatin or addon-statin extended-release nicotinic acid /laropiprant for the first four weeks followed by $2000 / 40 \mathrm{mg}$ /day for the next eight weeks OR to add-on-statin micronized fenofibrate $(200 \mathrm{mg} /$ day $)$ for a total of 3 months. Study findings showed that all treatment interventions reduced the assessed oxidative stress markers (27).

\section{Long-term and short-term therapy}

While assessing the role of combination (statin + fenofibrate) in long-term and short-term therapy of mixed dyslipidemia, a post hoc analysis was conducted. The results showed that in the controlled studies, remarkably reduced level of high-risk patients treated with fenofibric acid + moderate dose statin [rosuvastatin 10,20 or $40 \mathrm{mg}$, simvastatin 20 , 40 or $80 \mathrm{mg}$, or atorvastatin 20,40 or $80 \mathrm{mg}$ ] and a substantially higher percentage of high-risk patients treated with fenofibric acid and low dose statin compared with monotherapies were able to achieve their LDL-C (51.3\% vs. $72.9 \%, \mathrm{p}<0.001)$ and non-HDL$\mathrm{C}$ targets $(53 \%$ vs. $38 \%, \mathrm{p}<0.02)$, respectively. This was suggestive of the fact that short-term treatment with fenofibric acid and low or moderate dose statin led to a comparable or higher number of patients attaining individual targets of non-HDL-C, ApoB, HDL-C, and TG, and combined targets for these values and LDL-C, compared with corresponding monotherapy with any of the statins used in the study (rosuvastatin, simvastatin or atorvastatin). $(28,29)$ - Another long-term study conducted to evaluate the safety and efficacy of combination lipid therapy reported that fenofibric acid and a moderate-dose statin (Rosuvastatin $20 \mathrm{mg} /$ Atorvastatin $40 \mathrm{mg}$ / Simvastatin $40 \mathrm{mg}$ ) exhibited good tolerance and did not cause any new or unanticipated adverse reactions. This phase 3, open-label, year 2 extension study in patients who had completed one of the three doubleblind, 12-week, controlled studies and the subsequent open-label, year 1 extension study also showed that the combination lipid therapy, when used for over 2 years, led to a sustained improvement in HDLC (+17.4\%), TG (-46.4\%) and LDL-C (-40.4\%). 
Hence, long-term therapy resulted in elaborate and sustained lipid improvements in patients with mixed dyslipidemia (30).

\section{Safety and efficacy of rosuvastatin and fenofi- brate combination}

An acceptable safety profile of fenofibric acid and rosuvastatin has been established in a multicentre, randomized study. The study was conducted to evaluate the short-term efficacy and safety profile of fenofibric acid + rosuvastatin combination therapy for improving lipid parameters in patients with stage 3 chronic kidney disease and mixed dyslipidemia. Study findings showed that fenofibric acid $(45 \mathrm{mg})$ and rosuvastatin $(5 \mathrm{mg})$ compared with only rosuvastatin $5 \mathrm{mg}$ led to a significant improvement in triglycerides (median \% changes: week $8,-38.0 \%$ vs $-22 \%$, $\mathrm{P}<0.001$; week 16, $-42.6 \%$ vs-29.7\%, $\mathrm{P}<0.001)$ and HDL-C (mean \% changes: week $8,16.9 \%$ vs $7.8 \%$, $\mathrm{P}<0.001$; week $16,17.3 \%$ vs $8.9 \%, \mathrm{P}<0.001)(31)$.

In another study, following three months of treatment with the highest dose of rosuvastatin $(40 \mathrm{mg} /$ day $)$ or to add-on-statin extended-release nicotinic acid (ER-NA)/laropiprant (LRPT) or add-on-statin micronized fenofibrate, results revealed that add-on ER-NA/LRPT followed by switching to the highest dose rosuvastatin brought about significant benefits in emerging cardiovascular risk factors compared with add-on fenofibrate in patients with mixed dyslipidemia. However, another important finding was that ER-NA/LRPT was linked with more side effects than rosuvastatin and add-on fenofibrate (32).

In a study conducted among high-risk Asian patients with mixed hyperlipidemia, the rosuvastatinfenofibrate combination led to an incidence of myoor hepatotoxicity as compared with rosuvastatin monotherapy. Additionally, the combination group was associated with higher increases in homocysteine, blood urea nitrogen, serum creatinine, and much larger reductions in leukocyte and hemoglobin levels. However, it was suggested that the combination might require caution in individuals with underlying pathologies such as renal dysfunction. The study findings revealed that the combination group had higher, but not significantly, common treatment-related adverse events $(13.3 \%$ and $5.6 \%$. respectively) and drug discontinuation due to ad- verse events (10.0\% and 3.3\%, respectively) (33).

In a randomized clinical study, where 1,377 patients with mixed hyperlipidemia were treated with either fenofibric acid $135 \mathrm{mg}$, rosuvastatin 10,20 , or 40 $\mathrm{mg}$, or fenofibric acid plus rosuvastatin 10 or $20 \mathrm{mg}$, fenofibric acid plus rosuvastatin $20 \mathrm{mg}$ led to more significant improvements in triglyceride levels ($42.9 \%$ versus $-25.6 \%, \mathrm{p}<0.001)$ and HDL-c $(+19.0 \%$ versus $+10.3 \%, \mathrm{p}<0.001)$ compared with rosuvastatin $20 \mathrm{mg}$ monotherapy. A significantly higher LDL-C reduction $(-38.8 \%$ versus $-6.5 \%, \mathrm{p}<0.001)$ compared with fenofibric acid monotherapy was observed. The levels of hsCRP were significantly lowered with fenofibric acid plus rosuvastatin 10 or $20 \mathrm{mg}$ treatment compared with the corresponding dose of rosuvastatin monotherapy $(\mathrm{p}<0.05)$ (34)

In phase 3 , multicentre, randomized, double-blind study conducted on patients with mixed dyslipidemia with high low-density lipoprotein cholesterol, triglycerides, and low level of high-density lipoprotein cholesterol, the results revealed that the combination of rosuvastatin $5 \mathrm{mg} /$ day with fenofibric acid $135 \mathrm{mg}$ /day for 12 weeks in patients with mixed dyslipidemia led to a significant increase in HDL$\mathrm{C}$ plasma concentration $(+23.0 \%$ versus $+12.4 \%$, $\mathrm{p}<0.001)$ and a significant lowering of the TG levels $(-40.3 \%$ versus $-17.5 \%, \mathrm{p}<0.001)$ compared with rosuvastatin monotherapy. These results suggested that rosuvastatin $5 \mathrm{mg}+$ fenofibric acid $135 \mathrm{mg}$ led to substantial improvement in the lipid profile of the patients with mixed dyslipidemia and did not cause any unwarranted adverse effects (35). Similar results were obtained in phase 3 , multicentre, randomized, double-blind, active-controlled study, where the combination formulation of choline salt of fenofibric acid given with two doses of rosuvastatin showed that the combination therapy led to significantly $(\mathrm{p}<0.001)$ greater improvements in HDLC $(20.3 \%$ vs. $8.5 \%)$ and TG $(-47.1 \%$ vs. $-24.4 \%)$ compared to rosuvastatin $10 \mathrm{mg}$, and fenofibrate. Based on the above, the authors concluded that the combination caused a better optimization of many lipid parameters than monotherapy (34).

When assessed in patients with high low-density lipid and triglyceride levels, combination treatment with rosuvastatin/fenofibric acid was reported to 


\section{ROSUVASTATIN AND FENOFIBRATE COMBINATION IN THE TREATMENT OF MIXED HYPERLIPIDEMIA: A NARRATIVE REVIEW}

be well-tolerated. The results of this randomized, double-blind study also showed that the combination therapy with statin (rosuvastatin) and fibrate (fenofibric acid) doses led to a higher reduction in the low-density lipid-cholesterol and improved other efficacy parameters, compared with simvastatin 40 mg (36). An open-label, randomized, parallel-group, comparative, prospective clinical study was conducted to compare the effects of atorvastatin and rosuvastatin in combination with fenofibrate in patients with mixed hyperlipidemia. The results showed that patients who received a combination of atorvastatin $(10 \mathrm{mg})$ with fenofibrate $(160 \mathrm{mg})$ demonstrated a lowering of total cholesterol by $39 \%$, triglycerides by $47 \%$, LDL-C by $50 \%$, and VLD-C by $35 \%$. Simultaneously, in the rosuvastatin $(10 \mathrm{mg})$ and fenofibrate (160 mg) group, a reduction of total cholesterol occurred by $54 \%$, TGs by $58 \%$, LDL-C by $52 \%$, and VLDL-C by $56 \%$. The authors concluded that both the treatment regimens led to a considerable reduction in TC, TG, LDL-C, and VLDL-C; however, the reduction was more pronounced and statistically significant rosuvastatin fenofibrate combination group when compared with atorvastatin and fenofibrate after the 12-week treatment period (37).

\section{Effect of Rosuvastatin and Fenofibrate combina- tion on Potential cardiovascular disease markers}

Mixed dyslipidemia, oxidative stress, and inflammation are linked to an increased risk for cardiovascular disorders. Being a common metabolic disorder with notable features such as increased cholesterol and triglycerides presents a therapeutic challenge wherein monotherapy with statins may only be useful in the partial treatment of the underlying metabolic disorders. (38).

The mixed dyslipidemia phenotype comprises increased triglyceride levels, reduced high-density lipid cholesterol, and increased ApoB linked with diabetes and increased coronary artery disease risk. Fenofibric acid is commonly used to treat individuals with mixed dyslipidemia, and it decreases triglycerides, ApoB containing VLDL particles, and APOC-III while increasing HDL-C. A study showed that using a combination of fenofibric acid and statins in individuals with mixed dyslipidemia might cause an attenuated ApoB reduction if they are homozygous for the minor allele of single nucleotide polypeptide in both the ANGPTL3 and RXRA gene regions. This may lead to the diminution of cardiovascular risk $(39,40)$.

The low levels of $25(\mathrm{OH})$ vitamin $\mathrm{D}$ may be a novel risk factor for cardiovascular disease, the metabolic syndrome and other aspects associated with it (hypertension, atherogenic dyslipidemia, impaired glucose tolerance, and central obesity), diabetes mellitus, and even for cancer, autoimmune diseases, infections, and overall mortality (41). Results from many large, cross-sectional studies found an association of $25(\mathrm{OH})$ Vit D deficiency with an increased rate of myocardial infarction $(42,43)$. Initially, it was proposed that the cause for the rise in $25(\mathrm{OH})$ vitamin $\mathrm{D}$ levels in patients on statin combination therapy may be due to the competition in the cytochrome P450 3A4 (CYP3A4) catabolic pathway, which breaks down $25(\mathrm{OH})$ vitamin D levels (44). Another possible mechanism may be the inhibition of the 3hydroxy-3methylglutaryl coenzyme A (HMG-CoA) reductase by statins, which may lead to an increase in the 7-dehydrocholesterol levels, which in turn acts as the common precursor of cholesterol and 25 $(\mathrm{OH})$ vitamin $\mathrm{D}(45)$. In a randomized trial, high dose rosuvastatin monotherapy and the usual dose of rosuvastatin + fenofibrate or omega- 3 fatty acid was associated with a significant and similar increase in the $25(\mathrm{OH})$ vitamin D levels. In this study, 60 patients with dyslipidemia were randomly allocated to receive rosuvastatin $40 \mathrm{mg}$, rosuvastatin $10 \mathrm{mg}$ plus fenofibrate $200 \mathrm{mg}$, or rosuvastatin $10 \mathrm{mg}$ plus omega-3 fatty acids $2 \mathrm{~g}$ daily for three months. Rosuvastatin monotherapy led to a $53 \%$ increase in $25(\mathrm{OH})$ Vit D (from $14.6[1.0-38.0]$ to 17.8 [5.3$49.6] \mathrm{ng} / \mathrm{mL} ; \mathrm{P}=.000)$. The combination of rosuvastatin with fenofibrate and omega-3 fatty acids were related with increase of $64 \%$ (from 14.1 [1.0-48.0] to 18.4 [6.7-52.4] ng/mL; $\mathrm{P}=0.001$ ) and $61 \%$ (from 10.4 [6.6-38.4] to 14.0 [9.6-37.6] $\mathrm{ng} / \mathrm{mL} ; \mathrm{P}=0.04)$, respectively (41). The study results are important from a clinical perspective since low levels of 25 $(\mathrm{OH})$ Vit D have been identified as an independent CVD risk factor $(43,44)$.

The two hypolipidemic drugs also reduce Lipoprotein-associated phospholipase A2 (LpPLA2) activity and mass associated with atherogenic apoB-lipoproteins is a predictor for incident 


\section{CURRENT MEDICAL RESEARCH AND OPINION}

atherosclerotic disease. Additionally, fenofibrate was also found to improve the enzyme-specific activity on apoB-lipoproteins and induce HDL-LpPLA2 (46).

\section{4 | CONCLUSION}

Statins are established to be the standard-of-care therapy for lowering the low-density lipoprotein cholesterol; however, in patients with mixed dyslipidemia experiencing the abnormal 'lipid triad,' monotherapy alone becomes inadequate. In such cases, combining other lipid-modifying agents is needed to optimize lipid profiles in patients with mixed dyslipidemia. The combination therapy with fenofibric acid has been proven beneficial, welltolerated with a similar safety profile compared with statin monotherapy. The combination therapy of moderate dose rosuvastatin and fenofibric acid leads to a diminution of cardiovascular risk factors via several pathways.

Acknowledgments: The authors did not receive any funding from external or internal sources or assistance with the preparation of the manuscript. The authors have no conflict of interest to declare that they are relevant to this article's content.

Author contributions: Ajoy Tiwari (AT): Conception and design, literature search, data extraction of the relevant articles, drafting and critically revising the article, and final approval of the published version.

Kaushik Biswas (KB): Literature search, data extraction of the relevant articles, qualitative assessment of the eligible articles, drafting and critically revising the article, and final approval of the version to be published.

Prachi Jadhav (PJ): Critical review of the article, data extraction from the relevant article, and final approval of the version to be published.

Amit Goel (AG): Contributed towards conception and design, data extraction from the researched articles, qualitative assessment of the selected articles, critically revised the article, and final approval of the version to be published.

GV Chanukya (GVC): Contributed towards qualitative assessment of the selected articles, critically revised the article, and final approval of the version to be published.

\section{REFERENCES}

1. Kipnes MS, Roth EM, Rhyne JM, Setze CM, Lele A, Kelly MT, et al. Year Two Assessment of Fenofibric Acid and Moderate-Dose Statin Combination. Clinical Drug Investigation. 2010;30(1):51-61. Available from: https://dx. doi.org/10.2165/11319800-000000000-00000. doi:10.2165/11319800-000000000-00000.

2. Durrington PN, Tuomilehto J, Hamann A, Kallend D, Smith K. Rosuvastatin and fenofibrate alone and in combination in type 2 diabetes patients with combined hyperlipidaemia. Diabetes Research and Clinical Practice. 2004;64(2):137-151. Available from: https: //dx.doi.org/10.1016/j.diabres.2003.11.012. do i:10.1016/j.diabres.2003.11.012.

3. Makariou S, Liberopoulos EN, Elisaf M, Challa A. Novel roles of vitamin D in disease: What is new in 2011? European Journal of Internal Medicine. 2011;22(4):355-362. Available from: https://dx.doi.org/10.1016/j.ejim.2011.04.012. doi:10.1016/j.ejim.2011.04.012.

4. Roth EM, Rosenson RS, Jones PH, Davidson MH, Kelly MT, Setze CM, et al. Attainment of goal/desirable lipid levels in patients with mixed dyslipidemia after 12 weeks of treatment with fenofibric acid and rosuvastatin combination therapy: A pooled analysis of controlled studies. Journal of Clinical Lipidology. 2012;6(6):534544. Available from: https://dx.doi.org/10.1016/ j.jacl.2012.02.002. doi:10.1016/j.jacl.2012.02.0 02.

5. Strain JD, Farver D. A review on the rationale and clinical use of concomitant rosuvastatin and fenofibrate/fenofibric acid therapy. Clinical Pharmacology: Advances and Applications. 2010;2:95-95. Available from: https://dx.doi. org/10.2147/cpaa.s7375. doi:10.2147/cpaa.s737 5 . 
6. Mach F, Baigent C, Catapano AL, Koskinas KC, Casula M, Badimon L, et al. 2019 ESC/EAS Guidelines for themanagement of dyslipidaemias: lipid modification to reduce cardiovascular risk. Russian Journal of Cardiology. 2020;25(5):3826-3826. Available from: https: //dx.doi.org/10.15829/1560-4071-2020-3826. d oi:10.15829/1560-4071-2020-3826.

7. Lee SH, Cho KI, Kim JY, Ahn YK, Rha SW, Kim YJ. Non-lipid effects of rosuvastatinfenofibrate combination therapy in high-risk Asian patients with mixed hyperlipidemia. Atherosclerosis. 2012;221:169-75.

8. Mcgovern ME. Taking aim at HDL-C: Raising levels to reduce cardiovascular risk. Postgrad Med. 2005;117:29-44.

9. Wander GS, Moyk H, Yalagudri S, Mahajan B, Toppo A. Rosuvastatin: Role in Secondary Prevention of Cardiovascular Disease. JAPI. 2018;66.

10. Tziomalos K, Athyros VG. Fenofibrate: a novel formulation (Triglide?) in the treatment of lipid disorders: a review. International Journal of Nanomedicine. 2006;1(2):129-147. Available from: https://dx.doi.org/10.2147/nano.2006.1.2. 129. doi:10.2147/nano.2006.1.2.129.

11. Kei A, Liberopoulos E, Tellis K, Rizzo M, Elisaf M, Tselepis A. Effect of hypolipidemic treatment on emerging risk factors in mixed dyslipidemia: a randomized pilot trial. European Journal of Clinical Investigation. 2013;43(7):698707. Available from: https://dx.doi.org/10.1111/ eci.12095. doi:10.1111/eci.12095.

12. Agouridis AP, Kostapanos MS, Tsimihodimos V, Kostara C, Mikhailidis DP, Bairaktari ET, et al. Effect of rosuvastatin monotherapy or in combination with fenofibrate or $\omega-3$ fatty acids on lipoprotein subfraction profile in patients with mixed dyslipidaemia and metabolic syndrome. International Journal of Clinical Practice. 2012;66(9):843-853. Available from: https://dx.doi.org/10.1111/j.1742-1241.2012. 02972.x. doi:10.1111/j.1742-1241.2012.0297 2.x.
13. Jones PH, Davidson MH, Stein EA, Bays HE, McKenney JM, Miller E, et al. Comparison of the efficacy and safety of rosuvastatin versus atorvastatin, simvastatin, and pravastatin across doses $($ STELLAR $* *$ STELLAR $=$ Statin Therapies for Elevated Lipid Levels compared Across doses to Rosuvastatin. Trial). The American Journal of Cardiology. 2003;92(2):152160. Available from: https://dx.doi.org/10.1016/ s0002-9149(03)00530-7. doi:10.1016/s0002-91 49(03)00530-7.

14. Ferdinand KC, Davidson MH, Kelly MT, Setze $\mathrm{CM}$. One-year efficacy and safety of rosuvastatin + fenofibric acid combination therapy in patients with mixed dyslipidemia: Evaluation of dose-response. Am J Cardiovasc Drugs. 2012;12:117-142.

15. Kei A, Tellis C, Liberopoulos E, Tselepis A, Elisaf M. Effect of Switch to the Highest Dose of Rosuvastatin Versus Add-onStatin Fenofibrate Versus Add-on-Statin Nicotinic Acid/Laropiprant on Oxidative Stress Markers in Patients with Mixed Dyslipidemia. Cardiovascular Therapeutics. 2014;32(4):139146. Available from: https://dx.doi.org/10.1111/ 1755-5922.12072. doi:10.1111/1755-5922.120 72.

16. Makariou SE, Liberopoulos EN, Agouridis AP, Challa A, Elisaf M. Effect of Rosuvastatin Monotherapy and in Combination With Fenofibrate or Omega-3 Fatty Acids on Serum Vitamin D Levels. Journal of Cardiovascular Pharmacology and Therapeutics. 2012;17(4):382386. Available from: https://dx.doi.org/10.1177/ 1074248412439470. doi:10.1177/10742484124 39470.

17. Weinstein DL, Williams LA, Carlson DM, Kelly MT, Burns KM, Setze CM. A randomised, double-blind study of fenofibric acid plus rosuvastatin compared with rosuvastatin alone in stage 3 chronic kidney disease. Clin Ther. 2013;35:1186-98. 


\section{CURRENT MEDICAL RESEARCH AND OPINION}

18. Florentin M, Elisaf MS, Mikhailidis DP, Liberopoulos EN. Vitamin D and Metabolic Syndrome: Is There a Link? Current Pharmaceutical Design. 2010;16(30):3417-3434. Available from: https://dx.doi.org/10.2174/ 138161210793563509. doi:10.2174/13816121 0793563509.

19. Grandi NC, Breitling LP, Brenner H. Vitamin $\mathrm{D}$ and cardiovascular disease: Systematic review and meta-analysis of prospective studies. Preventive Medicine. 2010;51(3-4):228233. Available from: https://dx.doi.org/10.1016/ j.ypmed.2010.06.013. doi:10.1016/j.ypmed.201 0.06 .013 .

20. Agouridis AP, Rizos CV, Elisaf MS, Filippatos TD. Does Combination Therapy with Statins and Fibrates Prevent Cardiovascular Disease in Diabetic Patients with Atherogenic Mixed Dyslipidemia? The Review of Diabetic Studies. 2013;10(2-3):171-190. Available from: https:// dx.doi.org/10.1900/rds.2013.10.171. doi:10.190 0/rds.2013.10.171.

21. $\mathrm{Xu} \mathrm{Y,} \mathrm{Hashizume} \mathrm{T,} \mathrm{Shuhart} \mathrm{MC,} \mathrm{Davis} \mathrm{CL,}$ Nelson WL, Sakaki T, et al. Intestinal and Hepatic CYP3A4 Catalyze Hydroxylation of $1 \alpha, 25-$ Dihydroxyvitamin D3: Implications for DrugInduced Osteomalacia. Molecular Pharmacology. 2006;69(1):56-65. Available from: https: //dx.doi.org/10.1124/mol.105.017392. doi:10.1 124/mol.105.017392.

22. Rosenson RS, Carlson DM, Kelly MT, Setze CM, Hirshberg B, Stolzenbach JC, et al. Achievement of Lipid Targets with the Combination of Rosuvastatin and Fenofibric Acid in Patients with Type 2 Diabetes Mellitus. Cardiovascular Drugs and Therapy. 2011;25(1):4757. Available from: https://dx.doi.org/10.1007/ s10557-010-6273-5. doi:10.1007/s10557-010-6 273-5.

23. Roth EM, Rosenson RS, Carlson DM, Fukumoto SM, Setze CM, Blasetto JW, et al.. Efficacy and Safety of Rosuvastatin $5 \mathrm{mg}$ in Combination with Fenofibric Acid $135 \mathrm{mg}$ in $\mathrm{Pa}-$ tients with Mixed Dyslipidemia - A Phase 3
Study. Springer Science and Business Media LLC; 2010. Available from: https://dx.doi.org/ 10.1007/s10557-010-6266-4. doi:10.1007/s105 57-010-6266-4.

24. Grundy SM, Stone NJ, Bailey AL, Beam C, Birtcher KK, Blumenthal RS. AHA/ACC/AACVPR/AAPA/ABC/ACPM/ADA/AGS Guideline on the Management of Blood Cholesterol: Executive Summary: A Report of the American College of Cardiology/American Heart Association Task Force on Clinical Practice Guidelines. Circulation. 2018;139:1046-81.

25. Wilt TJ, Bloomfield HE, MacDonald R, Nelson D, Rutks I, Ho $M$, et al. Effectiveness of Statin Therapy in Adults With Coronary Heart Disease. Archives of Internal Medicine. 2004;164(13):1427-1427. Available from: https://dx.doi.org/10.1001/archinte.164. 13.1427. doi:10.1001/archinte.164.13.1427.

26. Agouridis AP, Tsimihodimos V, Filippatos TD, Tselepis AD, Elisaf MS. High Doses of Rosuvastatin are Superior to Low Doses of Rosuvastatin Plus Fenofibrate or n-3 Fatty Acids in Mixed Dyslipidemia. Lipids. 2011;46(6):521528. Available from: https://dx.doi.org/10.1007/ s11745-011-3538-0. doi:10.1007/s11745-011-3 538-0.

27. Ballantyne CM, Jones PH, Kelly MT, Setze CM, Lele A, Thakker KM, et al. Long-Term Efficacy of Adding Fenofibric Acid to ModerateDose Statin Therapy in Patients with Persistent Elevated Triglycerides. Cardiovascular Drugs and Therapy. 2011;25(1):59-67. Available from: https://dx.doi.org/10.1007/s10557011-6280-1. doi:10.1007/s10557-011-6280-1.

28. Ma L, Ballantyne CM, Belmont JW, Keinan A, Brautbar A. Interaction between SNPs in the RXRA and near ANGPTL3 gene region inhibits apoB reduction after statin-fenofibric acid therapy in individuals with mixed dyslipidemia. Journal of Lipid Research. 2012;53(11):24252428. Available from: https://dx.doi.org/10. 1194/j1r.m028829. doi:10.1194/j1r.m028829. 
29. Kendrick J, Targher G, Smits G, Chonchol M. 25-Hydroxyvitamin D deficiency is independently associated with cardiovascular disease in the Third National Health and Nutrition Examination Survey. Atherosclerosis. 2009;205(1):255-260. Available from: https: //dx.doi.org/10.1016/j.atherosclerosis.2008.10. 033. doi:10.1016/j.atherosclerosis.2008.10.033.

30. Jones PH, Davidson MH, Kashyap ML, Kelly MT, Buttler SM, Setze CM, et al. Efficacy and safety of ABT-335 (fenofibric acid) in combination with rosuvastatin in patients with mixed dyslipidemia: A phase 3 study. Atherosclerosis. 2009;204(1):208-215. Available from: https: //dx.doi.org/10.1016/j.atherosclerosis.2008.09. 027. doi:10.1016/j.atherosclerosis.2008.09.027.

31. Pappa E, Rizos CV, Filippatos TD, Elisaf MS. Emerging Fixed-Dose Combination Treatments for Hyperlipidemia. Journal of Cardiovascular Pharmacology and Therapeutics. 2019;24(4):315-322. Available from: https://dx. doi.org/10.1177/1074248419838506. doi:10.11 77/1074248419838506.

32. Ford ES, Giles WH, Dietz WH. Prevalence of the Metabolic Syndrome Among US Adults: Findings From the Third National Health and Nutrition Examination Survey. Obstetrical \& Gynecological Survey. 2002;57(9):576577. Available from: https://dx.doi.org/10.1097/ 00006254-200209000-00017. doi:10.1097/000 06254-200209000-00017.

33. Dixit R, Jagan S. Comparative Study of Atorvastatin and Rosuvastatin in Combination with Fenofibrate in mixed Hyperlipidemia. International Journal of Pharmacology and Clinical Sciences. 2016;5(1):25-31. Available from: https:// dx.doi.org/10.5530/ijpcs.5.1.5. doi:10.5530/ijpc s.5.1.5.

34. Agouridis AP, Tsimihodimos V, Filippatos TD, Dimitriou AA, Tellis CC, Elisaf MS, et al. The effects of rosuvastatin alone or in combination with fenofibrate or omega 3 fatty acids on inflammation and oxidative stress in patients with mixed dyslipidemia. Expert Opinion on Pharmacotherapy. 2011;12(17):26052611. Available from: https://dx.doi.org/10.
1517/14656566.2011.591383. doi:10.1517/146 56566.2011 .591383$.

35. Pepine CJ, Jacobson TA, Carlson DM, Kelly MT, Setze CM, Gold A, et al. Combination Rosuvastatin Plus Fenofibric Acid in a Cohort of Patients 65 Years or Older With Mixed Dyslipidemia: Subanalysis of Two Randomized, Controlled Studies. Clinical Cardiology. 2010;33(10):609-619. Available from: https:// dx.doi.org/10.1002/clc.20830. doi:10.1002/clc. 20830.

36. Martin PD, Dane AL, Schneck DW, Warwick MJ. An open-label, randomized, threeway crossover trial of the effects of coadministration of rosuvastatin and fenofibrate on the pharmacokinetic properties of rosuvastatin and fenofibric acid in healthy male volunteers. Clinical Therapeutics. 2003;25(2):459471. Available from: https://dx.doi.org/10.1016/ s0149-2918(03)80089-9. doi:10.1016/s0149-29 18(03)80089-9.

37. Sattar N, Gaw A, Scherbakova O, Ford I, O'Reilly DSJ, Haffner SM, et al. Metabolic Syndrome With and Without C-Reactive Protein as a Predictor of Coronary Heart Disease and Diabetes in the West of Scotland Coronary Prevention Study. Circulation. 2003;108(4):414419. Available from: https://dx.doi.org/10.1161/ 01.cir.0000080897.52664.94. doi:10.1161/01.ci r.0000080897.52664.94.

38. Cleeman JI. Executive summary of the third report of the National Cholesterol Education Program (NCEP) expert panel on detection, evaluation, and treatment of high blood cholesterol in adults (adult treatment panel III). JAMA. 2001;285:2486-97.

39. Roth EM, McKenney JM, Kelly MT, Setze CM, Carlson DM, Gold A, et al. Efficacy and Safety of Rosuvastatin and Fenofibric Acid Combination Therapy versus Simvastatin Monotherapy in Patients with Hypercholesterolemia and Hypertriglyceridemia. American 


\section{CURRENT MEDICAL RESEARCH AND OPINION}

Journal Cardiovascular Drugs. 2010;10(3):175186. Available from: https://dx.doi.org/10.2165/ 11533430-000000000-00000. doi:10.2165/115 33430-000000000-00000.

40. Detection, evaluation, and treatment of high blood cholesterol in adults (Adult Treatment Panel III). Rev Panam Salud Publica. 2001;9:338-382.

41. Makariou SE, Liberopoulos EN, Agouridis AP, Challa A, Elisaf M, Effect of rosuvastatin monotherapy and in combination with fenofibrate or omega-3 fatty acids on serum vitamin D levels, J Cardiovasc Pharmacol Ther. 17(2012) 382-6. Available from: https://pubmed.ncbi.nl m.nih.gov/22431864/;

42. Saougos VG, Tambaki AP, Kalogirou M, Kostapanos M, Gazi IF, Wolfert RL, et al. Differential Effect of Hypolipidemic Drugs on Lipoprotein-Associated Phospholipase A 2. Arteriosclerosis, Thrombosis, and Vascular Biology. 2007;27(10):2236-2243. Available from: https://dx.doi.org/10.1161/atvbaha. 107.147280. doi:10.1161/atvbaha.107.147280.

43. Zhu T, Awni WM, Hosmane B, Kelly MT, Sleep DJ, Stolzenbach JC, et al. ABT-335, the Choline Salt of Fenofibric Acid, Does Not Have a Clinically Significant Pharmacokinetic Interaction With Rosuvastatin in Humans. The Journal of Clinical Pharmacology. 2009;49(1):6371. Available from: https://dx.doi.org/10.1177/ 0091270008325671. doi:10.1177/00912700083 25671.
44. Ballantyne CM, Davidson MH, Setze CM, Kelly MT. Effects of combination therapy with rosuvastatin and fenofibric acid in patients with mixed dyslipidemia and high-sensitivity Creactive protein $(\geq 2 \mathrm{mg} / \mathrm{L})$. Journal of Clinical Lipidology. 2011;5(5):401-407. Available from: https://dx.doi.org/10.1016/j.jacl.2011.06. 010. doi:10.1016/j.jacl.2011.06.010.

45. Mohiuddin SM, Thakker KM, Setze CM, Kelly MT. Evaluating optimal lipid levels in patients with mixed dyslipidemia following short- and long-term treatment with fenofibric acid and statin combination therapy: a post hoc analysis. Current Medical Research and Opinion. 2011;27(5):1067-1078. Available from: https: //dx.doi.org/10.1185/03007995.2011.569018. d oi:10.1185/03007995.2011.569018.

46. Guryev O, Carvalho RA, Usanov S, Gilep A, Estabrook RW. A pathway for the metabolism of vitamin D3: Unique hydroxylated metabolites formed during catalysis with cytochrome P450sce (CYP11A1). Proceedings of the National Academy of Sciences; 2003. Available from: https://dx.doi.org/10.1073/pnas. 2336107100. doi:10.1073/pnas.2336107100.

How to cite this article: Tiwari A., Biswas K., Jadhav P., Goel A., Chanukya G.V. Rosuvastatin and Fenofibrate Combination in The Treatment of Mixed Hyperlipidemia: A Narrative Review. Journal of Current Medical Re-search and Opinion. 2021;867-877.

https://doi.or g/10.15520/jcmro.v4i03.405 